\title{
Solving the wind farm layout optimization problem using random search algorithm
}

\author{
Feng, Ju; Shen, Wen Zhong
}

Published in:

Renewable Energy

Link to article, DOI:

10.1016/j.renene.2015.01.005

Publication date:

2015

Document Version

Early version, also known as pre-print

Link back to DTU Orbit

Citation (APA):

Feng, J., \& Shen, W. Z. (2015). Solving the wind farm layout optimization problem using random search algorithm. Renewable Energy, 78, 182-192. https://doi.org/10.1016/j.renene.2015.01.005

\section{General rights}

Copyright and moral rights for the publications made accessible in the public portal are retained by the authors and/or other copyright owners and it is a condition of accessing publications that users recognise and abide by the legal requirements associated with these rights.

- Users may download and print one copy of any publication from the public portal for the purpose of private study or research.

- You may not further distribute the material or use it for any profit-making activity or commercial gain

- You may freely distribute the URL identifying the publication in the public portal

If you believe that this document breaches copyright please contact us providing details, and we will remove access to the work immediately and investigate your claim. 


\title{
Solving the Wind Farm Layout Optimization Problem Using Random Search Algorithm
}

\author{
Ju Feng, Wen Zhong Shen \\ Technical University of Denmark, Department of Wind Energy, DK-2800 Lyngby, Denmark \\ jufen@dtu.dk wzsh@dtu.dk
}

\begin{abstract}
Wind farm (WF) layout optimization is to find the optimal positions of wind turbines (WTs) inside a WF, so as to maximize and/or minimize a single objective or multiple objectives, while satisfying certain constraints. In this work, a random search (RS) algorithm based on continuous formulation is presented, which starts from an initial feasible layout and then improves the layout iteratively in the feasible solution space. It was first proposed in our previous study and improved in this study by adding some adaptive mechanisms. It can serve both as a refinement tool to improve an initial design by expert guesses or other optimization methods, and as an optimization tool to find the optimal layout of WF with a certain number of WTs. A new strategy to evaluate layouts is also used, which can largely save the computation cost. This method is first applied to a widely studied ideal test problem, in which better results than the genetic algorithm (GA) and the old version of the RS algorithm are obtained. Second it is applied to the Horns Rev $1 \mathrm{WF}$, and the optimized layouts obtain a higher power production than its original layout, both for the real scenario and for two constructed scenarios. In this application, it is also found that in order to get consistent and reliable optimization results, up to 360 or more sectors for wind direction have to be used. Finally, considering the inevitable inter-annual variations in the wind conditions, the robustness of the optimized layouts against wind condition changes is analyzed, and the optimized layouts consistently show better performance in power production than the original layout, despite of considerable variations in wind direction and speed.
\end{abstract}

Keywords: wind farm layout optimization, random search, refinement tool, optimization tool, Horns Rev, robustness.

\section{Introduction}

A wind farm is a group of WTs located at a site to generate electricity, which is also called as "plant", "cluster", "array" and "park" in literature. The world's first onshore WF was installed in 1980 on the shoulder of Crotched Mountain in southern New Hampshire, USA, with a capacity of 0.6 MW, consisting of 20 WTs with a rated power of $30 \mathrm{~kW}$ [1]. In 1991, the world's first offshore WF, Vindeby offshore WF was erected off the north coast of the Danish Island Lolland, which marked the beginning of offshore wind energy. It has a total capacity of $4.95 \mathrm{MW}$ consisting of 11 Bonus 450 $\mathrm{kW}$ WTs [2]. Nowadays, the progress of technologies, such as power electronics [3], wind speed forecasting [4], coordinated control [5], together with the increased experience of WF construction and operation have enabled the development of modern WFs, i.e., larger, smarter WFs, which are typically consisting of hundreds of utility-scale (multi-MW) WTs and with a total capacity of hundreds MW. In parallel with this trend, the efforts for increasing the percentage of wind power in the total electricity consumption have led to the proliferation of modern WFs.

Due to the multi-disciplinary nature and the evolution towards larger size, smarter control and more advanced capabilities, the development of WF is becoming a highly complex process which pursues multiple and in many cases conflicting objectives under different constraints. It involves different design and engineering tasks, which may come from technical, logistical, environmental, economical, legitimacy and even social considerations [6].

Among all these tasks, the optimization of WF layout is a critical one. In literature, WF layout usually refers to the placement of WTs inside a certain area. Therefore, WF layout optimization is to determine the positions of WTs inside the WF in maximizing and/or minimizing some objective functions, such as maximizing the energy production and 
minimizing the cost, while meeting various constraints, which may include WF boundary, WTs proximity, noise emission level, initial investment limit, and so on. In general cases, i.e., considering the selection of WT number, WT type, discrete hub height, WF layout optimization is a multi-objective mixed integer-discrete-continuous nonlinear constrained optimization problem without analytical formulation. It is mathematically complex and can't be solved by using classical analytical optimization techniques.

In the last two decades, this complex problem has received more and more attention. Different problem formulations have been proposed and various optimization algorithms have been used to tackle this problem. Previous works are based on various simplified formulations, such as array of equally spaced turbines [7,8], array of unequally spaced turbines [9], aligned or staggered grid like (row-column) layout [10,11], pre-divided discrete grid points [12], continuous searching space [13,14], using a range of algorithms, such as Monte Carlo [8,15], GA [12,16], simulated annealing (SA) [14], particle swarm optimization (PSO) [17] and local search algorithm [18]. Different kinds of objectives are used, e.g., maximizing the power [9], annual energy production (AEP) [14, 18], profit [8,13], net present value (NPV) [11], or minimizing the cost of energy (CoE) [12, 15-17], levelized production cost (LPC) [19]. More comprehensive survey of published works can be found in several papers [20-22].

The random search (RS) algorithm for WF layout optimization in our previous study [23] was based on a continuous formulation and used to refine results obtained by GA [16] for an ideal test problem [12]. In this study, the algorithm is improved by adding some adaptive mechanisms and applied first for the same ideal test problem and then for the Horns Rev 1 WF. To save the computation cost, a strategy to evaluate the layouts, which is similar with that adopted by Wagner et al. in [18], is also applied. It is found that the improved method can achieve better optimization results than its old version for the ideal test problem with the same number of evaluations. For the Horns Rev 1 WF, it also can improve the power production by using optimized layouts. Besides, the pre-processing of wind resource data and the robustness of the obtained layouts against wind change are discussed in this paper. In the meanwhile, the same algorithm was used in a preliminary study to optimize a WF layout in complex terrain and obtained steady improvements over expert guess layouts for a WF on an ideal Gaussian shape hill [24].

\section{Problem formulation}

\subsection{Wind modeling}

Appropriate wind modeling is the starting point for accurate predicting wind farm power production. In Mosetti's study [12], three simple wind cases were constructed and applied to his test problem. Although it is valid to use this kind of ideal wind modeling when focusing on algorithm investigation, more realistic wind modeling is required for real-life application.

To assess the wind resource at a planned wind farm site, a wind measurement campaign is usually first carried out at a reference height $H_{\text {ref }}$. The obtained measurement data can be processed by using method of bins [25], and then written in matrix form as

$$
\boldsymbol{F}=\left(F_{w k}\right), \text { with } w=1,2, \ldots, N_{w s}, k=1,2, \ldots, N_{w d}
$$

where $F_{w k}=p_{o c c}\left(v_{w}, \theta_{k}\right)$ denotes the frequency of occurrence of wind speed $v_{w}$ in direction $\theta_{k} ; N_{w s}$ and $N_{w d}$ are number of bins for wind speed and for wind direction, respectively. Furthermore, the summarized wind data can be fitted sector-wisely into a certain probability distribution, typically Weibull distribution, which is governed by

$$
p_{W b}\left(v, A_{k}, c_{k}\right)=\left(\frac{c_{k}}{A_{k}}\right)\left(\frac{v}{A_{k}}\right)^{c_{k}-1} \exp \left[-\left(\frac{v}{A_{k}}\right)^{c_{k}}\right]
$$

Then the wind resource data can be presented as: $\left[\theta_{k}, A_{k}, c_{k}, f_{k}\right]$ with $k=\left[1,2, \ldots, N_{w d}\right]$, i.e., in terms of: direction angle, scale factor, shape factor and frequency of occurrence. In order to predict the power production, the inflow wind speed $V_{0}$ at hub height $H$ should be calculated, usually using the logarithmic law: 
where $v_{w}$ denotes the wind speed at reference height $H_{r e f}, z_{0}$ is the surface roughness length. Wind modeling form of Eq. (1) is convenient for numerical calculation due to its discrete nature. When the wind data is given in the form of Weibull distribution, it can also be easily converted into a matrix form by discretization. If the directional sector is too wide, i.e., $N_{w d}$ is too small, each sector can also be further divided into smaller sub-sectors with same scale factor $A_{k}$ and shape factor $c_{k}$.

94

\subsection{Wake modeling}

In order to calculate the wind field in WFs, the wake effects between WTs have to be modeled appropriately. Due to the nature of optimization problems, a quite large number of layout evaluations must be carried out, which requires simple and reliable wake modeling. In most of the layout optimization works in literature and also in some commercial software such as WAsP, the Jensen wake model [26], also known as PARK wake model or Katic wake model, is used. This model is developed by assuming that momentum is conserved within the wake, and the wake region expands linearly in the direction of wind flow.

Suppose there are $N_{w t}$ WTs in the WF and the layout is represented by $\boldsymbol{X}=\left[x_{1}, x_{2}, \ldots, x_{N_{w t}}\right], \quad \boldsymbol{Y}=\left[y_{1}\right.$, $\left.y_{2}, \ldots, y_{N_{w t}}\right]$. Considering WT $i$ at location $\left(x_{i}, y_{i}\right)$ and WT $j$ at location $\left(x_{j}, y_{j}\right)$ for wind direction $\theta_{k}$, the original Cartesian coordinates can first be rotated according to $\theta_{k}$ so that wind blows along the new $x^{\prime}$ direction. If $x_{i}^{\prime} \leq x_{j}^{\prime}$, WT $j$ is at the downwind of WT $i$ or at the same level, and therefore have no influence on WT $i$. If $x_{i}^{\prime}>x_{j}^{\prime}$, wind speed and wake zone radius behind WT $j$ and at the position where WT $i$ is located, denoted as $V_{i j}$ and $R_{i j}$, are governed by the following expressions:

$$
\begin{gathered}
V_{i j}=V_{0}\left[1-\frac{1-\sqrt{1-C_{T}\left(V_{0}\right)}}{\left(1+\alpha\left(x_{i j}^{\prime} / R_{r}\right)\right)^{2}}\right], \\
R_{i j}=\alpha \cdot x_{i j}^{\prime}+R_{r}
\end{gathered}
$$

where $V_{0}$ is the inflow wind speed, $C_{T}\left(V_{0}\right)$ denotes the thrust coefficient of WT at wind speed $V_{0}, \alpha$ is the wake decay coefficient, $R_{r}=D / 2$ represents the radius of rotor and $x_{i j}^{\prime}=x_{i}^{\prime}-x_{j}^{\prime}$ is the distance between the two WTs along wind direction. Besides, the affected area of WT $i$ 's rotor by WT $j$ 's wake is calculated as the overlapping area of two circles with radiuses $R_{r}, R_{i j}$ and centres distanced at $\left|y_{i j}^{\prime}\right|=\left|y_{i}^{\prime}-y_{j}^{\prime}\right|$, or zero when it's not in the downstream of WT $j$, i.e.,

$$
A_{i j}= \begin{cases}A_{o l}\left(R_{r}, R_{i j},\left|y_{i j}^{\prime}\right|\right), & x_{i}^{\prime}>x_{j}^{\prime}, \\ 0, & x_{i}^{\prime} \leq x_{j}^{\prime} .\end{cases}
$$

The formula for calculating $A_{o l}$ and its derivation are given in the Appendix. Note that $A_{i j} / A_{r}$ is used as an effective percentage for the wake effect of WT $j$ on WT $i$, where $A_{r}=\pi R_{r}^{2}$ is the rotor area.

Based on the mutual wake effects between any two WTs described in Eqs. (4-6), the effective wind speed WT $i$ experienced can be derived based on the kinetic energy deficit balance assumption, as

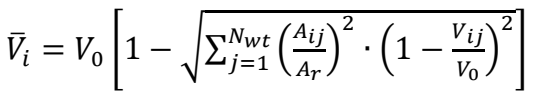

Substituting Eq. (4) into Eq. (7), it can be rewritten as

$$
\bar{V}_{i}=V_{0}\left[1-\left(1-\sqrt{1-C_{T}\left(V_{0}\right)}\right) \cdot \sqrt{\sum_{j=1}^{N_{w t}}\left(\frac{A_{i j}}{A_{r}}\right)^{2} /\left[1+\alpha\left(x_{i j}^{\prime} / R_{r}\right)\right]^{4}}\right]
$$

We can define the wake influence factor of WT $j$ on WT $i$ along wind direction $\theta_{k}$ as

$$
M_{i j k}=\left(\frac{A_{i j}}{A_{r}}\right)^{2} /\left[1+\alpha\left(x_{i j}^{\prime} / R_{r}\right)\right]^{4}
$$


Then the wake influence tensor of the wind farm, denoted as $\boldsymbol{M}=\left(M_{i j k}\right)$ is a function of the layout $(\boldsymbol{X}, \boldsymbol{Y})$. Therefore, for a given layout, the wake influence tensor $\boldsymbol{M}$ can be initialized. During the optimization process, if only the $i$ th WT is moved, $\boldsymbol{M}$ can be updated by re-calculating $\boldsymbol{M}(i,:,:)$ and $\boldsymbol{M}(:, i,:)$ while the other parts remain unchanged. Note that this strategy can largely save the computation cost when evaluating different layouts in the optimization process [18].

\subsection{Power production}

For a given type of WTs, the power curve $=P(v)$, thrust curve $C_{T}=C_{T}(v)$, cut-in wind speed $V_{\text {in }}$, cut-out wind speed $V_{\text {out }}$ are usually provided by the manufacturer, which can be used to calculate the power production of wind farm. Considering Eq. (3) and Eq. (8) and noticing that the wake modeling presented above is derived for a given wind direction $\theta_{k}$, one can see that $\bar{V}_{i}$ in Eq. (8) is a function of reference wind speed $v_{w}$, wind direction $\theta_{k}$ and WF layout $(\boldsymbol{X}, \boldsymbol{Y})$, i.e., $\bar{V}_{i}=\bar{V}_{i}\left(v_{w}, \theta_{k}, \boldsymbol{X}, \boldsymbol{Y}\right)$. Using the frequency of occurrence of wind speed $v_{w}$ in direction $\theta_{k}$, i.e., $F_{w k}=$ $p_{\text {occ }}\left(v_{w}, \theta_{k}\right)$, the expected power production by WF is derived as

$$
P_{t o t}=\sum_{i=1}^{N_{w t}} \sum_{k=1}^{N_{w d}} \sum_{w=1}^{N_{w s}} P\left(\bar{V}_{i}\left(v_{w}, \theta_{k}, \boldsymbol{X}, \boldsymbol{Y}\right)\right) \cdot F_{w k}
$$

Substitute Eq. (8), it can rewritten as

$$
\left.P_{t o t}=\sum_{i=1}^{N_{w t}} \sum_{k=1}^{N_{w d}} \sum_{w=1}^{N_{w s}} P\left(v^{H}\left(v_{w}\right)\left[1-\left(1-\sqrt{1-C_{T}\left(v^{H}\left(v_{w}\right)\right.}\right)\right) \times \sqrt{\sum_{j=1}^{N_{w t} M_{i j k}}}\right]\right) \cdot F_{w k}
$$

Note that for a given wind resource $\boldsymbol{F}$ and a given type of WTs, $P_{t o t}$ of a WF with a fixed number of WTs depends only on the layout $(\boldsymbol{X}, \boldsymbol{Y})$, i.e., it can be denoted as $P_{\text {tot }}=P_{\text {tot }}(\boldsymbol{X}, \boldsymbol{Y})$.

Similarly, the ideal power production of the same WF without wake effect can be easily obtained as

$$
P_{\text {ideal }}=N_{w t} \times\left[\sum_{k=1}^{N_{w d}} \sum_{w=1}^{N_{w s}} P\left(v^{H}\left(v_{w}\right)\right) \cdot F_{w k}\right]
$$

and the WF efficiency is defined as

$$
\eta=P_{\text {tot }} / P_{\text {ideal }}
$$

\subsection{Constraints and assumptions}

WF development is subject to various constraints, which may come from technical, logistical, environmental, economical, legitimacy or even social consideration. In the context of layout optimization, two constraints are commonly considered. One is the boundary of WF, the other is minimal distance between WTs. For a WF with irregular shape and/or with infeasible zone inside the boundary, the boundary constraint can be dealt by the single-/multiboundary model incorporated with the ray intersection method proposed by Gu et al. [27]. In this study, the boundary is assumed to be a polygonal, with the feasible zone of WT's locations defined as a polyhedron:

$$
S_{\text {feasible }}=\left\{(x, y) \mid a_{k} x+b_{k} y \leq c_{k}, k=1, \ldots, m\right\}
$$

And the boundary constraint can be expressed as

$$
\left(x_{i}, y_{i}\right) \in S_{\text {feasible }}, \quad \text { for } i=1,2, \ldots, N_{w t}
$$

Since close distance between WTs will introduce large wake loss and high turbulence intensity, which in turn increases the fatigue of blades and shorten the lifetime of WTs, it is usually required to place the WTs according to the minimal distance constraint. The constraint can be provided by WT manufacturers and also depends on the specific terrain feature and wind resource characteristics at the given site. In this study, it is simply assumed to be a minimal distance requirement between any two WTs, governed by

$$
\sqrt{\left(x_{i}^{2}-x_{j}^{2}\right)+\left(y_{i}^{2}-y_{j}^{2}\right)} \geq \text { Dist }_{\text {min }}, \quad \text { for } i, j=1,2, \cdots, N_{w t} \text { and } i \neq j
$$

There are also some underlying assumptions in the above WF modeling, including: (1) all WTs are of the same type, i.e., with same rotor size, power curve and thrust curve; (2) all WTs are installed with the same hub height; (3) the terrain of WF is flat; (4) the wind resource inside the WF is uniform. Besides, in the power production calculation, only the wake loss is taken into consideration. Other factors such as availability factor, electrical loss, icing loss, may also 
affect the power production. Although electrical loss may partially depend on the WF layout, most of these factors are not affected by the layout, and therefore are not included in the present modeling.

\subsection{Objective function}

The most widely used objective for WF layout optimization is to minimize the cost of energy, which represents the cost per unit of produced energy. Although not strictly correct in its physical meaning, the cost of energy (CoE) defined by Mosetti [12], i.e., cost of the WF divided by the total power production, is widely used in literature:

$$
\mathrm{CoE}=\mathrm{Cost} / P_{\text {tot }}
$$

where 'Cost' represent the cost of the WF, modeled by a simple function which only depends on the number of WTs:

$$
\text { Cost }=N_{w t}\left(\frac{2}{3}+\frac{1}{3} e^{-0.00174 N_{w t}^{2}}\right)
$$

Since in the RS algorithm used in this study, the number of WTs is fixed, it is easily seen from Eqs. (17-18) that minimizing ' $\mathrm{CoE}$ ' is identical to maximizing ' $P_{\text {tot }}$ '. Therefore, the optimization problem in this study can be stated as: choose a WF layout of $N_{w t}$ WTs to maximize the total power production, i.e.,

$$
\max P_{\text {tot }}=P_{\text {tot }}(\boldsymbol{X}, \boldsymbol{Y})
$$

while the layout $(\boldsymbol{X}, \boldsymbol{Y})$ is subject to the constraints given in Eqs. (14-16).

\section{RS algorithm}

In the previous study [23], a RS algorithm for WF layout optimization was proposed based on continuous formulation and applied to an ideal test problem in flat terrain [12]. It was shown that the RS algorithm can obtain better optimization results comparing to GA. The algorithm starts from an initial feasible layout and then improves the layout iteratively in the feasible solution space according to the objective and constraints. When moving the WTs during the search process, the old version of RS algorithm selects one WT randomly and moves its position randomly at each 'Random Move' step [23], here this process is improved by adding some adaptive mechanisms, i.e., remembering and utilizing the information of a good move, which means a 'Random Move' step that results in power increase. The procedure of the improved RS algorithm is shown in Algorithm 1.

Algorithm 1: Random search (RS) algorithm for wind farm layout optimization

\section{Initialize:}

Select initial layout $\mathbf{S}_{\mathbf{0}}$ (from the optimized layout using other algorithms, expert guesses or from a random feasible layout);

Evaluate objective function: $\mathbf{f}_{0}=\mathbf{f}\left(\mathbf{S}_{\mathbf{0}}\right)$; Set Improve_flag $=$. .False..

While stop condition is not true:

\section{Random Move}

If (Improve_flag $==$.False.):

Select a WT randomly, move its position in a random direction with a random step:

$\mathbf{S}=\mathbf{S}_{\mathbf{0}}+\Delta \mathbf{S}$. (Note: $\Delta \mathbf{S}$ is bounded by the long edge of the WF)

Else:

Select the WT moved last time, move along the old direction, with a random step size.

\section{End If}

\section{Feasibility Check}

Check feasibility of $\mathbf{S}$ using constraints of the problem

If $\mathrm{S}$ is not feasible:

Repeat the Random Move (step 1)

\section{End If}




\title{
3. Layout Evaluation
}

Calculate the objective function of feasible layout $\mathbf{S}: \mathbf{f}=\mathbf{f}(\mathbf{S})$

(Note: In this step, since only $1 \mathrm{WT}$ is moved, updating the wake influence matrix $\mathbf{M}$ and then calculating the power of WF will save a large amount of efforts)

\section{Optimal Layout Update If $(\mathbf{f}>\mathbf{f 0})$ :}

\author{
Set $\mathbf{S}_{0}=\mathbf{S}, \mathbf{f}_{0}=\mathbf{f}$, Improve_flag $=$.True..
}

Else:

Set Improve_flag $=$.False..

\section{End If}

\section{End While}

So is the optimized layout

It should be noticed that there is a 'Feasibility Check' step in the algorithm, which deals with the constraints of the problem, i.e., the WF boundary constraint and minimal distance constraint given in Eqs. (14-16). As the algorithm tries to move a WT randomly in the 'Random Move' step, it is not guaranteed that all the constraints will be satisfied for the new layout, therefore, this explicit feasibility check is necessary. The other feature needs to be pointed out is that the number of WTs is fixed in this algorithm, which stays the same as in the initial layout.

This algorithm is simple, intuitive and easy to implement. It can be used as a last stage refinement tool to improve the results obtained by any other algorithms, or as an optimization tool to optimize the layout of wind farm with a given number of WTs. If the number of WTs is not specified, the layout optimization problem can be solved by running RS algorithm multiple times with a different number of WTs at each time and then finding the overall best layout. One should also note that this algorithm employs randomness to search for better solutions and the process is stopped after a certain number of evaluations [28]. Therefore, it usually obtains different results in different runs for the same problem. So it is often necessary to run the algorithm for multiple times and study its performance variation.

\section{Ideal test problem}

To evaluate the effectiveness of the improved RS algorithm, it is first applied to solve an ideal test problem, which was proposed by Mosetti et al. in their seminal work [12], and later widely studied in literature [16]. In our previous study [23], the original RS algorithm was used to refine the layouts obtained by GA in [16] for this ideal test problem. Similarly the improved RS algorithm is first applied to refine the GA results as in [23], then to optimize the layout of a WF with a fixed number of WTs by using random layouts as initial layouts. Three wind cases are considered: Case (1): uniform north wind with a speed of $12 \mathrm{~m} / \mathrm{s}$; Case (2): Equally distributed (36 directions) wind with a speed of $12 \mathrm{~m} / \mathrm{s}$; Case (3): Non-uniformly distributed (36 directions) wind with speeds of 8,12 and $17 \mathrm{~m} / \mathrm{s}$. The details of the wind cases and problem setting are referred to [12].

The performance of the optimized layouts for 3 wind cases are presented in Table 1, including comparisons with those obtained by GA [16] and old version of RS algorithm [23]. In this table, 'N' represents the number of WTs; 'GA Pub.' denotes the published results in [16], and 'GA Re-eval.' means the re-evaluated results of the obtained layouts using our code, 'Discrep.' represents the discrepancy of power between these two sets of values, which can be attributed to the difference in detailed wake modeling implementation, and especially the exact data of wind case (3) (data used here is extracted from the figure in [16]); 'RS-new 1' and 'RS-new 2' denote results obtained by the improved RS algorithm using GA results as initial layouts and random layouts as initial layouts, respectively; and 'Imp.' is the 
improvement percentages of the optimized layouts to the GA results (the re-evaluated values). It is worth to note that, for each case the presented result from RS is from the best run in 100 runs and each run uses maximally 100000 evaluations. The statistics of multiple runs are given in Table 2 .

It can be seen from Table 1 that the proposed algorithm can obtain steady improvements when starting from the layouts obtained by GA. When starting from random layouts, the optimized layouts have similar or better performance as the refined layouts from GA results, which suggests that the proposed algorithm can be used as an optimization tool for WF with a fixed number of WTs, without the need to seed it with good initial layouts. Consistent with the previous study, the greatest improvements are achieved for the wind case (1), which may be attributed to the smallest number of WTs and the single direction wind characteristic.

Table 1: Layout performance of the RS optimized results for the ideal test problem

\begin{tabular}{c|cccc|cccc|cccc}
\hline & \multicolumn{4}{|c|}{ Case (1) } & \multicolumn{4}{c|}{ Case (2) } & \multicolumn{4}{c}{ Case (3) } \\
& $\mathrm{N}$ & $\mathrm{CoE}$ & $\mathrm{P}(\mathrm{kW})$ & $\eta(\%)$ & $\mathrm{N}$ & $\mathrm{CoE}$ & $\mathrm{P}(\mathrm{kW})$ & $\eta(\%)$ & $\mathrm{N}$ & $\mathrm{CoE}$ & $\mathrm{P}(\mathrm{kW})$ & $\eta(\%)$ \\
\hline \hline GA Pub. & 30 & 0.001544 & 14310 & 92.0 & 39 & 0.001567 & 17220 & 85.2 & 39 & 0.000803 & 32038 & 86.6 \\
GA Re-eval. & 30 & 0.001544 & 14304 & 92.0 & 39 & 0.001568 & 17172 & 84.9 & 39 & 0.000851 & 31621 & 85.4 \\
\hline Discrep. (\%) & \multicolumn{6}{|c|}{0.04} & & \multicolumn{2}{|c|}{0.28} & & & \multicolumn{3}{c}{1.30} & \\
\hline \hline RS-old & 30 & 0.001454 & 15193 & 97.7 & 39 & 0.001547 & 17406 & 86.1 & 39 & 0.000839 & 32096 & 86.7 \\
\hline Imp. (\%) & $/$ & -5.83 & 6.22 & 6.22 & $/$ & -1.33 & 1.36 & 1.36 & $/$ & -1.41 & 1.50 & 1.50 \\
\hline \hline RS-new 1 & 30 & 0.001452 & 15209 & 97.8 & 39 & 0.001522 & 17691 & 87.5 & 39 & 0.000826 & $\mathbf{3 2 6 1 0}$ & 88.1 \\
\hline Imp. (\%) & $/$ & -5.96 & 6.33 & 6.33 & $/$ & -2.93 & 3.02 & 3.02 & $/$ & -2.94 & 3.13 & 3.13 \\
\hline \hline RS-new 2 & 30 & 0.001451 & $\mathbf{1 5 2 2 4}$ & 97.9 & 39 & 0.001521 & $\mathbf{1 7 6 9 7}$ & 87.5 & 39 & 0.000826 & 32581 & 88.0 \\
Imp. (\%) & $/$ & -6.02 & 6.43 & 6.43 & $/$ & -3.00 & 3.06 & 3.06 & $/$ & -2.94 & 3.04 & 3.04 \\
\hline
\end{tabular}

The original and optimized layouts when using initial layouts from GA results are shown in Figure 1 for all the three wind cases. Although all the optimized layouts produce higher power than their original layouts obtained by GA, no obvious pattern can be found from these layouts, except the trend that more WTs are located near the WF boundary for the wind cases with multiple wind directions, i.e., Case (2) and Case (3).
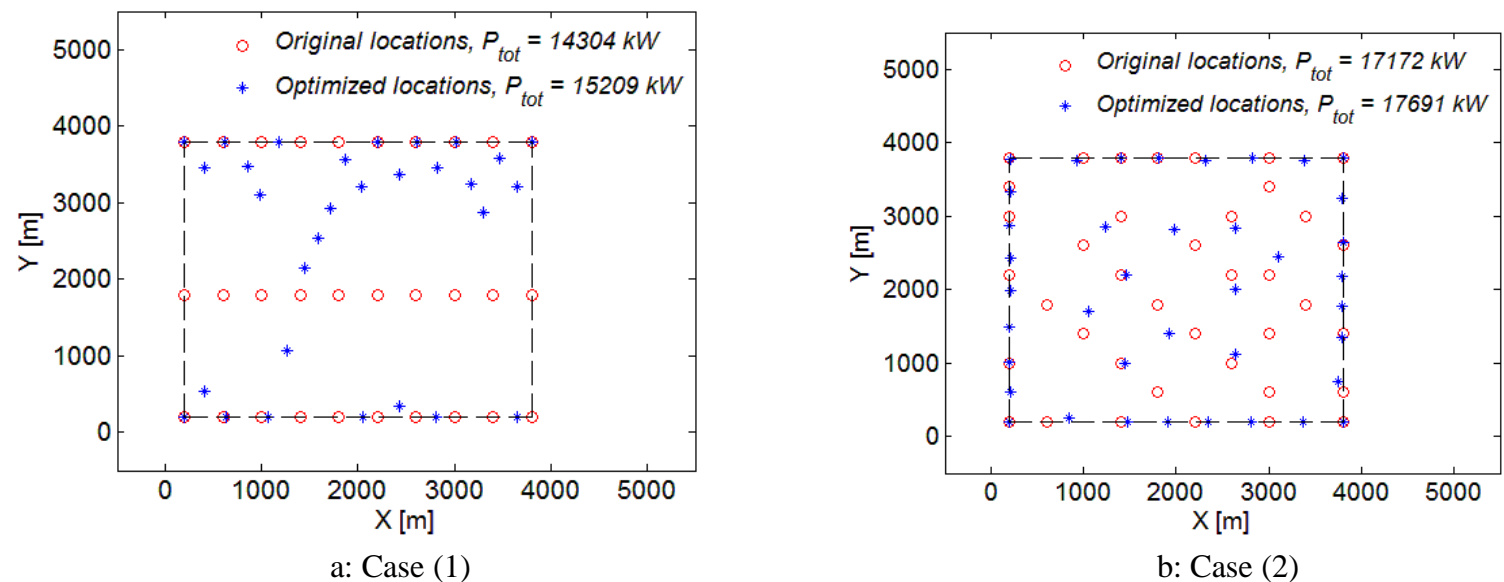

b: Case (2) 


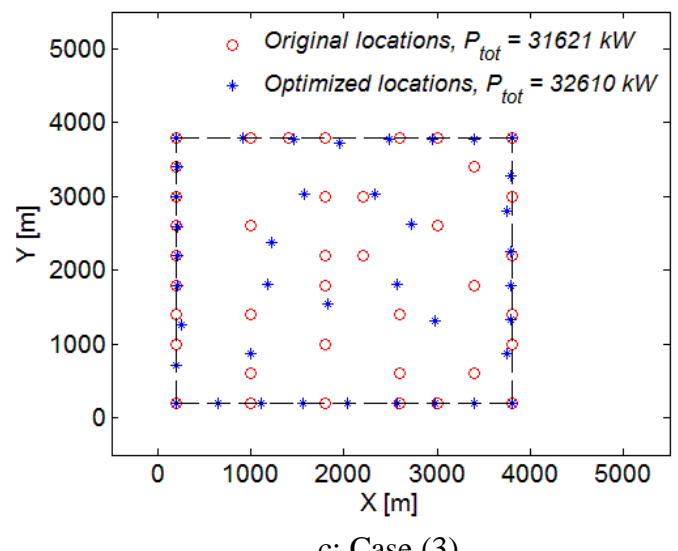

Figure 1: Original and optimized layouts for the ideal test problem (3 wind cases)

For this ideal test problem, the optimization processes are carried out in 100 different runs for each of the three cases, with maximal allowed evaluation number for each run set as 100000 . The statistics of these multiple runs are calculated and shown in Table 2. Note that the 'CPU time per run' presented in this paper, as in Table 2 and Table 5, is the average value from multiple runs, obtained by implementing the algorithm in Fortran 95 on a Dell@ laptop with an Intel@ i5-2520M CPU @ 2.50GHz.

Table 2: Performance variation of the RS algorithm in 100 runs for the ideal test problem

\begin{tabular}{|c|c|c|c|c|c|c|c|c|c|c|}
\hline & & Cas & $(1)$ & & Cas & (2) & \multicolumn{4}{|c|}{ Case (3) } \\
\hline \multicolumn{11}{|c|}{ RS-new 1 (using GA results as initial layouts) } \\
\hline \multirow{2}{*}{$\begin{array}{c}\text { Statistics of } \\
100 \text { runs }\end{array}$} & \multicolumn{10}{|c|}{$\mathrm{P}(\mathrm{kW})$} \\
\hline & $\begin{array}{c}\text { Best } \\
15209\end{array}$ & $\begin{array}{l}\text { Worst } \\
15085\end{array}$ & $\begin{array}{cc}\text { Mean } & \text { Std } \\
15148 & 22.8\end{array}$ & $\begin{array}{c}\text { Best } \\
17691\end{array}$ & $\begin{array}{l}\text { Worst } \\
17571\end{array}$ & $\begin{array}{cc}\text { Mean } & \text { Std } \\
17638 & 21.8\end{array}$ & $\begin{array}{c}\text { Best } \\
32610\end{array}$ & $\begin{array}{l}\text { Worst } \\
32391\end{array}$ & $\begin{array}{l}\text { Mean } \\
32497\end{array}$ & $\begin{array}{l}\text { Std } \\
44.8\end{array}$ \\
\hline CPU time per run & \multicolumn{3}{|c|}{$0.83 \mathrm{~s}$} & \multicolumn{3}{|c|}{$37.37 \mathrm{~s}$} & \multicolumn{4}{|c|}{$38.29 \mathrm{~s}$} \\
\hline \multicolumn{11}{|c|}{ RS-new 2 (using random layouts as initial layouts) } \\
\hline \multirow{2}{*}{$\begin{array}{c}\text { Statistics of } \\
100 \text { runs }\end{array}$} & \multicolumn{10}{|c|}{$\mathrm{P}(\mathrm{kW})$} \\
\hline & $\begin{array}{c}\text { Best } \\
15224\end{array}$ & $\begin{array}{l}\text { Worst } \\
15093\end{array}$ & $\begin{array}{cc}\text { Mean } & \text { Std } \\
15161 & 25.1\end{array}$ & $\begin{array}{c}\text { Best } \\
17697\end{array}$ & $\begin{array}{l}\text { Worst } \\
17558\end{array}$ & $\begin{array}{cc}\text { Mean } & \text { Std } \\
17630 & 27.5\end{array}$ & $\begin{array}{c}\text { Best } \\
32581\end{array}$ & $\begin{array}{l}\text { Worst } \\
32352\end{array}$ & $\begin{array}{l}\text { Mean } \\
32462\end{array}$ & $\begin{array}{c}\text { Std } \\
55.0\end{array}$ \\
\hline CUP time per run & \multicolumn{3}{|c|}{$0.92 \mathrm{~s}$} & \multicolumn{3}{|c|}{$38.91 \mathrm{~s}$} & \multicolumn{4}{|c|}{$39.88 \mathrm{~s}$} \\
\hline
\end{tabular}

It can be seen that the robustness of the RS algorithm is quite good when examining the standard deviation (std) of power in multiple runs, which means we can expect that the RS algorithm will probably achieve a quite satisfying performance with enough evaluations in all the cases. Also the largest improvements are achieved for the one wind direction case, which suggests that the existence of a single prevailing wind direction generally brings a larger improving space for WF layout optimization. Since the different runs of 'RS-new 2' start from different random layouts, while those of 'RS-new 1' start from the same GA obtained layouts, larger variations are seen for 'RS-new 2'. It is also shown that 'RS-new 2' obtains better optimization results from the best run in multiple runs in Case (1) and Case (2).

\section{Horns Rev 1 wind farm}

The Horns Rev 1 offshore WF is located about $15 \mathrm{~km}$ off the westernmost point of Denmark, which has a rated capacity of $160 \mathrm{MW}$ and consists of 80 Vestas V80 WTs (with a rotor diameter $D=80 \mathrm{~m}$ and a hub height $H=70 \mathrm{~m}$ ). The 
WTs are arranged in a grid manner inside a parallelogram area, with spacing along two main directions of $7 D$. Using data extracted from [29], the layout of the WF and the characteristics of the WTs are shown in Figure 2.
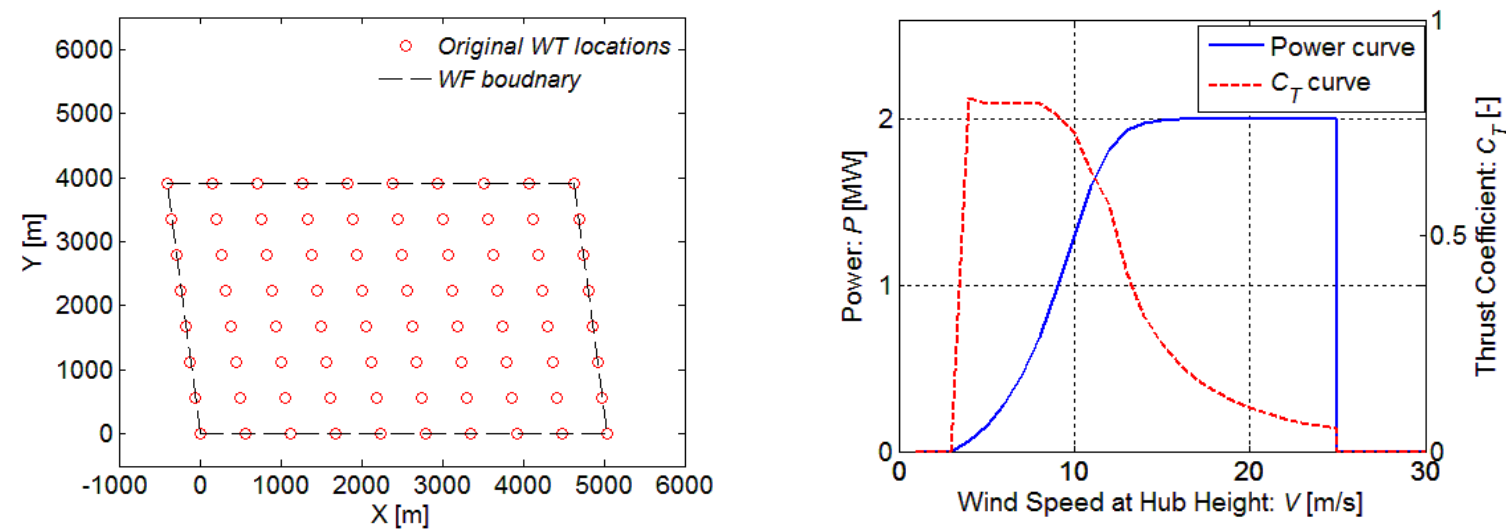

Figure 2: Original layout of the Horns Rev 1 WF and characteristic curves of a Vestas V80 WT

The wind resource data from January 1989 to October 2002 is extracted from Table 4.2 in [30], which presents the corrected Weibull parameters in 12 sectors for Horns $\operatorname{Rev}\left(H_{\text {ref }}=62 \mathrm{~m}\right)$. The data is shown in Table 3 and used in this study as the long term average wind resource for estimating the power production. In order to investigate the influence of different kinds of wind resource on the layout optimization problem, a new wind case with a strong single prevailing wind direction $\left(300^{\circ}\right)$ is constructed by adapting the frequencies of occurrence of different wind directions. Parameters of both wind cases are shown in Table 3. Note that in this table, $0^{\circ}, 90^{\circ}, 180^{\circ}, 270^{\circ}$ represents north, east, south and west wind, respectively, while other angles denote other directions in between.

Table 3: Measured and constructed wind cases for the Horns Rev 1 wind farm

\begin{tabular}{c|ccccccccccccc}
\hline Direction & $0^{\circ}$ & $30^{\circ}$ & $60^{\circ}$ & $90^{\circ}$ & $120^{\circ}$ & $150^{\circ}$ & $180^{\circ}$ & $210^{\circ}$ & $240^{\circ}$ & $270^{\circ}$ & $300^{\circ}$ & $330^{\circ}$ \\
\hline & \multicolumn{10}{c}{ Measured wind case } \\
\hline$A$ & 8.71 & 9.36 & 9.29 & 10.27 & 10.89 & 10.49 & 10.94 & 11.23 & 11.93 & 11.94 & 12.17 & 10.31 \\
$C$ & 2.08 & 2.22 & 2.41 & 2.37 & 2.51 & 2.75 & 2.61 & 2.51 & 2.33 & 2.35 & 2.58 & 2.01 \\
$\%$ & 3.8 & 4.3 & 5.5 & 8.3 & 8.7 & 6.7 & 8.4 & 10.5 & 11.4 & 12.2 & 13.9 & 6.1 \\
\hline \hline & \multicolumn{10}{|c}{ Constructed wind case } \\
\hline$A$ & 8.71 & 9.36 & 9.29 & 10.27 & 10.89 & 10.49 & 10.94 & 11.23 & 11.93 & 11.94 & 12.17 & 10.31 \\
$C$ & 2.08 & 2.22 & 2.41 & 2.37 & 2.51 & 2.75 & 2.61 & 2.51 & 2.33 & 2.35 & 2.58 & 2.01 \\
$\%$ & 5.0 & 5.0 & 5.0 & 5.0 & 5.0 & 5.0 & 5.0 & 5.0 & 5.0 & 5.0 & 45.0 & 5.0 \\
\hline
\end{tabular}

As shown in Figure 2, the WF boundary is set as the parallelogram according to the real layout, which can be described by constraint equations in the form of Eq. (14) and the minimal distance between any two WTs is set as Dist $_{\min }=5 \mathrm{D}$. Then the RS algorithm is applied to optimize the layout of $80 \mathrm{WTs}$ in order to produce a higher power than the original layout of Horns Rev 1 WF.

\subsection{Preprocessing wind resource data}

It is well known that the power production of a large WF is quite sensitive to wind direction due to the complex wake effects inside the WF. In their numerical study of the Horns Rev 1 WF [31], Porte-Agel et al. have showed that a change in wind direction of $10^{\circ}$ from the worst-case full-wake condition will increase the power production by as much as $43 \%$. 
Although this kind of dramatic change only occurs in certain wind directions and the sensitivity to wind direction is not so profound when considering all directions, it is still an important task to preprocess wind resource data appropriately in order to reduce the impact of wind direction uncertainty and increase the accuracy of power calculation. Given the measured wind data, it is then important to decide how many sectors of wind direction and how many bins of wind speed should be used.

The wind resource data used here is given in terms of Weibull parameters for 12 sectors, which can be further discretized into more sectors by dividing one sector into multiple sub-sectors with the same scale factor $A$ and shape factor $c$. In order to get consistent and reliable results in layout optimization, a certain number of sectors will be needed. On the other hand, using too many sectors of wind direction will increase the computation cost unnecessarily. Here, the options of using 12, 72 and 360 sectors, i.e., with sector widths of $30^{\circ}, 5^{\circ}$ and $1^{\circ}$ respectively, are tested. For the measured wind case shown in Table 3, wind data preprocessed with 3 options is obtained and then used in the layout optimization. The original layout of the Hos Rev $1 \mathrm{WF}$ is used as an initial layout, and the RS algorithm is run with 100000 evaluations for each option. Then the original and optimized layouts are re-evaluated in terms of power production using the same measured wind case preprocessed with a different number of sectors. The re-evaluated relative improvement in power production as a function of the number of sectors in re-evaluation is shown in Figure 3 for the 3 preprocessing options. The obtained improvement values in the 3 optimization processes are shown with stars in the figure.

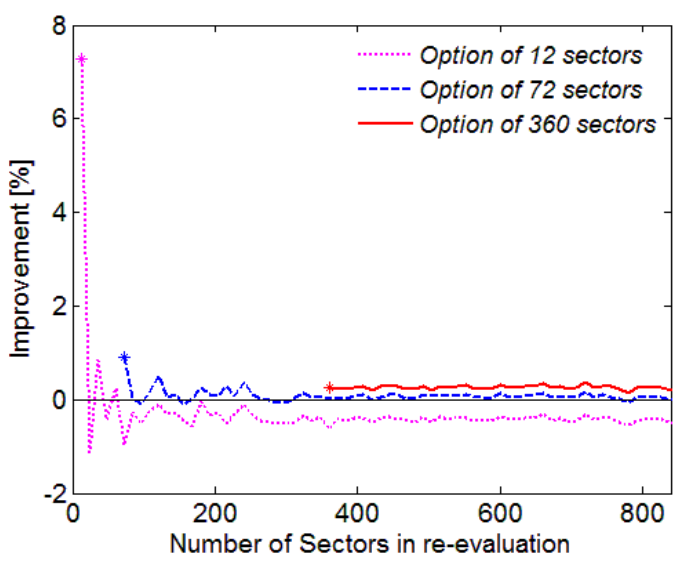

Figure 3: Power improvements of optimized layouts with 3 preprocessing options

(re-evaluated with wind data discretized into a larger number of sectors)

It can be seen that, when using 12 sectors the power is increased by impressively more than 7\%, but when reevaluated with finer discretization of wind direction, it actually decreases in power production. This phenomenon can be explained by the fact that using 12 sectors is assuming that wind only comes from 12 specific directions, while wind in reality comes from all directions. The discretization of wind direction leaves a large space for optimization algorithms to adjust the WF layout. Therefore, the results obtained by optimization using 12 sectors are unrealistic and should not be used for real-life applications. When using 72 sectors, the difference between the obtained value and the re-evaluated value (using more sectors) of the improvement is smaller than that of using 12 sectors, but it also shows a negative value when re-evaluated using more sectors. The achieved improvement by using 360 sectors is much smaller than those by using fewer sectors, but the result is much more consistent when re-evaluated using more sectors, i.e., finer discretization of wind direction. Therefore the result obtained by using 360 sectors is more consistent and reliable for real-life applications, although it looks not so impressive. Note that the oscillating behaviors shown in Figure 3 do not disappear with increasing the number of sectors in re-evaluation, this might be explained by the fact that the wind resource data used here is given in only 12 sectors and then further discretized, which still introduces high discontinuity 
between the neighboring sectors. When using raw data from wind measurement and preprocessed with an appropriate method, this oscillating behavior might be avoided.

Considering the results shown in the test study, the option of 360 sectors is chosen to preprocess the wind resource data, i.e., dividing the original 12 sectors into 360 sectors.

\subsection{Layout optimization}

For the Horns Rev 1 WF, 3 layout optimization cases are constructed: Case (1), measured wind case (as shown in Table 3) and original WF boundary (as shown in Figure 2); Case (2), constructed wind case (as shown in Table 3) and original WF boundary; Case (3), measured wind case and enlarged WF boundary (by enlarging the boundary with 7D in two main directions of the parallelogram, as shown in Figure 4e). The RS algorithm is applied to optimize the layout of the WF with 80 WTs for the 3 cases, either using the original layout, or using a random layout as the initial layout.

The performance of the optimized and the original layouts for the 3 cases is presented in Table 4. In this table, 'Original' denotes the original Horns Rev 1 WF, which serves as a baseline case; 'RS-new 1' and 'RS-new 2' denote results obtained by the RS algorithm using the original layout and a random layout as the initial layout, respectively. Also notice that for each case the presented result from RS is from the best run in 40 runs and each run uses maximally 100000 evaluations. The statistics of multiple runs are given in Table 5.

For the original scenario of the Horn Rev 1WF, i.e., Case (1), the optimized layout increases the power production by $0.37 \%$. When a strong single prevailing wind direction exists, as in Case (2), a larger improvement by $1.03 \%$ is achieved. When the WF boundary is enlarged, as in Case (3), the power production is increased by $1.53 \%$. Comparing these 3 cases, the existence of a strong prevailing wind direction is seen to be advantageous for WF layout optimization, as one can expect that a wind case homogenous in direction and in speed would prefer a homogenous layout, such as the original grid layout of the Horns Rev 1 WF. Enlarging the WF boundary will bring more improvement space for layout optimization, which may achieve higher power production. On the other hand, larger WF area usually means higher cost, because of increased internal network cost, land leasing or purchasing cost, building and maintenance cost and so on. Therefore, layout optimization of WFs in real life has to be carried out not just with power production consideration, but also with accurate cost considerations, various constraints and requirements. Also it can be seen that using random layout as initial layout achieves the best layout in Case (1) and Case (3), but in Case (2) using original layout outperforms.

Table 4: Performance of the RS optimized layouts for the Horns Rev 1 test problem

\begin{tabular}{c|cc|cc|cc}
\hline & \multicolumn{2}{|c|}{ Case (1) } & \multicolumn{2}{c|}{ Case (2) } & \multicolumn{2}{c}{ Case (3) } \\
& $\mathrm{P}(\mathrm{MW})$ & $\eta(\%)$ & $\mathrm{P}(\mathrm{MW})$ & $\eta(\%)$ & $\mathrm{P}(\mathrm{MW})$ & $\eta(\%)$ \\
\hline Original & 81.6720 & 92.6238 & 84.8004 & 93.0781 & 81.6720 & 92.6238 \\
\hline \hline RS-new 1 & 81.8927 & 92.8741 & $\mathbf{8 5 . 6 7 6 8}$ & 94.0401 & 82.9027 & 94.0196 \\
\hline Imp. (\%) & 0.2701 & 0.2701 & 1.0335 & 1.0335 & 1.5068 & 1.5068 \\
\hline \hline RS-new 2 & $\mathbf{8 1 . 9 7 7 0}$ & 92.9697 & 85.6086 & 93.9652 & $\mathbf{8 2 . 9 2 0 2}$ & 94.0394 \\
\hline Imp. (\%) & 0.3733 & 0.3733 & 0.9531 & 0.9531 & 1.5282 & 1.5282 \\
\hline
\end{tabular}

The optimized layouts against the original layout are shown in Figure 4. From the figure there is no obvious pattern found in these optimized layouts, except the trend that more WTs are re-located near the WF boundary. 


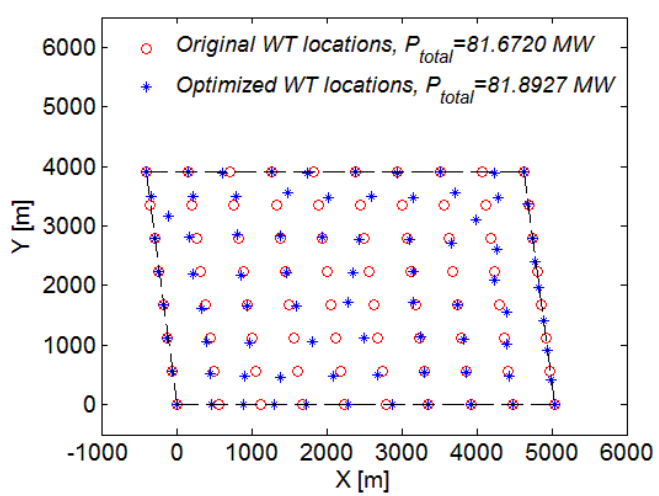

a: Case (1) from the original layout

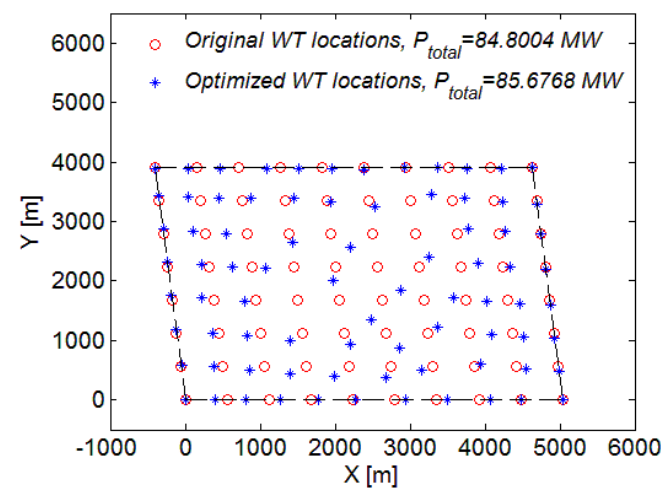

c: Case (2) from the original layout

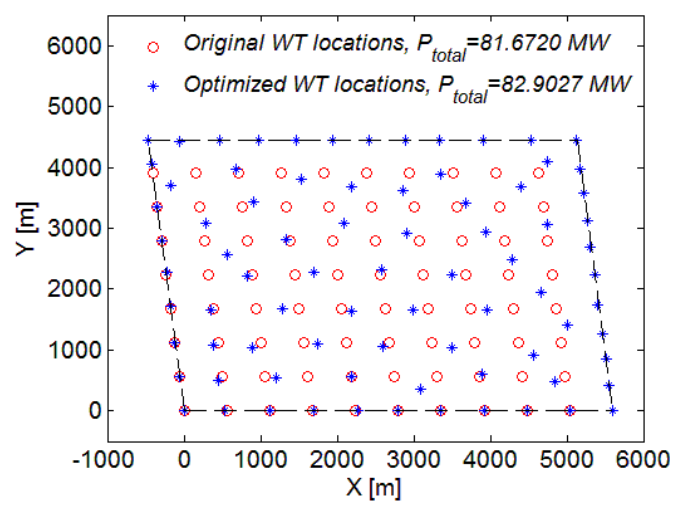

e: Case (3) from the original layout

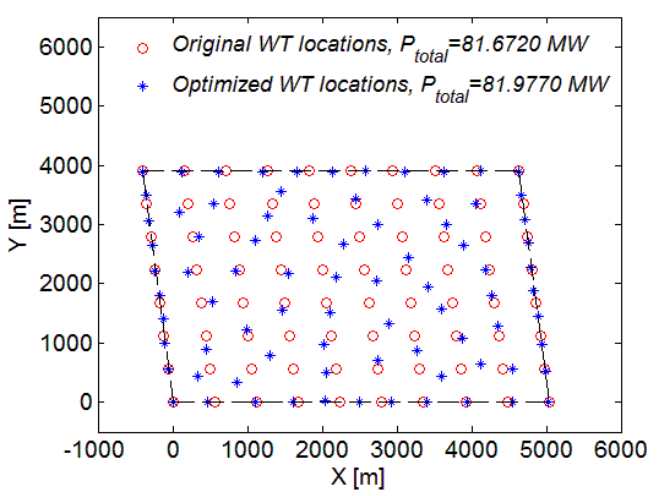

b: Case (1) from a random layout

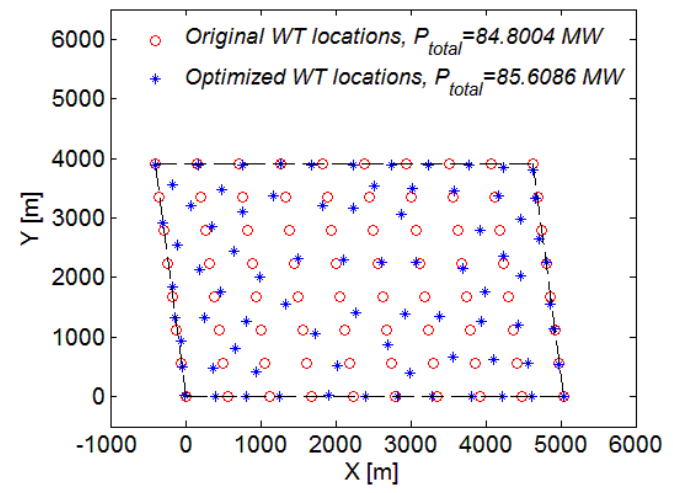

d: Case (2) from a random layout

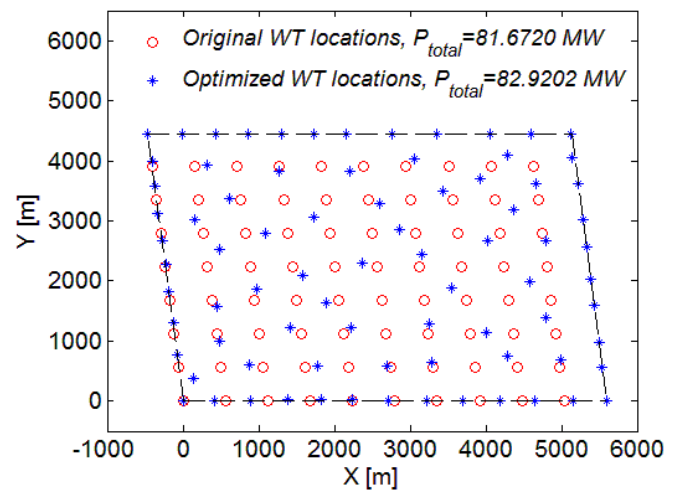

f: Case (3) from a random layout

Figure 4: Original and optimized layouts for the Horns Rev 1 test problem 


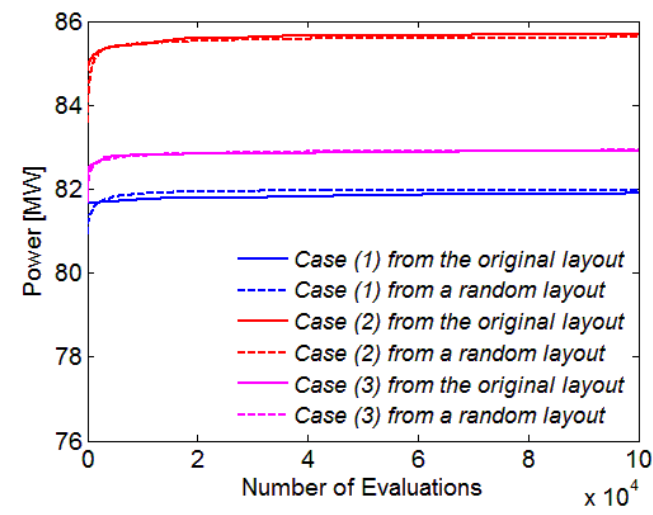

Figure 5: Evolution histories of the optimization processes

Comparing the evolution histories of the optimization process in different cases as shown in Figure 5, it can be seen that in the initial stage, i.e., with less than 10000 evaluations, the layout from the original layout achieves higher power production than that from a random layout and also the rapidest and largest improvement is achieved in this stage. In the latter stage, the increasing in power becomes slower and the differences between the results from the two kind of initial layouts become smaller. Observing this, one can conclude that the importance of seeding the optimization process with a good quality initial layout is more profound when using a small number of evaluations, while the difference is not obvious when using a large number of evaluations.

One can notice from Table 1 and Table 4 that the best achieved results in different cases are sometimes by RS-new 1, and sometimes by RS-new2, but the differences between the results of the two methods are not so big. This can be attributed to the random nature of this kind of meta-heuristics [28]. In practice, the choice of using RS-new 1 or RS-new 2 should be made based on the available computation budget. In other words, if the computation budget allows a large number of evaluations (up to 100000), it is not important to use good initial layouts, otherwise, it is advised to seed the optimization with good initial layouts, which may be obtained from expert guess or other pre-stage optimization process.

Note that the results presented above are from the best run out of 40 different runs for each case. The statistics of power production obtained in multiple runs are calculated and shown in Table 5. It can be seen that the RS algorithm achieves a quite robust performance in multiple runs. The variations of different runs are smaller for the runs starting from the original layout than those from random layouts, which is easy to understand since in one case it always starts from the same initial layout and in the other case it starts from different initial layouts for each run.

Table 5: Performance variation of the RS algorithm in 40 runs for the Horns Rev 1 test problem

\begin{tabular}{|c|c|c|c|c|c|c|c|c|c|c|c|c|}
\hline & & Cas & (1) & & & Cas & (2) & & \multicolumn{4}{|c|}{ Case (3) } \\
\hline \multicolumn{13}{|c|}{ RS-new 1 (using the original layout as initial layout) } \\
\hline \multirow{3}{*}{$\begin{array}{l}\text { Statistics of } \\
\text { multiple runs }\end{array}$} & \multicolumn{12}{|c|}{$\mathrm{P}(\mathrm{MW})$} \\
\hline & Best & Worst & Mean & Std & Best & Worst & Mean & Std & Best & Worst & Mean & Std \\
\hline & 81.89 & 81.79 & 81.83 & 0.021 & 85.68 & 85.58 & 85.62 & 0.022 & 82.90 & 82.81 & 82.85 & 0.022 \\
\hline CPU time per run & \multicolumn{4}{|c|}{$3498.25 \mathrm{~s}$} & \multicolumn{4}{|c|}{$3321.31 \mathrm{~s}$} & \multicolumn{4}{|c|}{$3215.02 \mathrm{~s}$} \\
\hline \multicolumn{13}{|c|}{ RS-new 2 (using a random layout as initial layout) } \\
\hline \multirow{3}{*}{$\begin{array}{l}\text { Statistics of } \\
\text { multiple runs }\end{array}$} & \multicolumn{12}{|c|}{$\mathrm{P}(\mathrm{MW})$} \\
\hline & Best & Worst & Mean & Std & Best & Worst & Mean & Std & Best & Worst & Mean & Std \\
\hline & 81.98 & 81.86 & 81.93 & 0.029 & 85.61 & 85.46 & 85.54 & 0.036 & 82.92 & 82.81 & 82.87 & 0.025 \\
\hline CPU time per run & \multicolumn{4}{|c|}{$3170.21 \mathrm{~s}$} & \multicolumn{4}{|c|}{$3485.69 \mathrm{~s}$} & \multicolumn{4}{|c|}{$3221.19 \mathrm{~s}$} \\
\hline
\end{tabular}




\subsection{Robustness analysis of the optimized layouts}

The temporal variations of wind can be classified into 4 scales: inter-annual, annual, diurnal, short-term (gusts and turbulence) [24]. Since WF layout optimization usually involves the evaluation of the energy yield from a WF during its 20 year lifetime, the inter-annual and annual variations are essential to consider. While the annual variations are well characterized by the Weibull distribution, there is no reliable prediction model that can be used to account the uncertainty brought by the inter-annual variations. Since the power production is strongly related to the wind conditions and the inter-annual wind variations commonly exist in different sites, the projected power production of a WF in its lifetime will also be inevitably affected. Therefore, it is important to assess the robustness of optimized layouts against long-term wind variations in terms of both wind direction and wind speed.

In order to assess the superiority of the optimized layout over the original layout, we have to consider not only the wind resource data from past measurements, but also wind resource data with possible long-term variations. In this section, the optimized layouts obtained for Case (1) and the original layout (as shown in Figure 4a and $4 \mathrm{~b}$ ) are evaluated with 3 types of changing wind resources: rotating the wind rose by $-20^{\circ}$ to $20^{\circ}$; changing the scale factors in all sectors by $-20 \%$ to $20 \%$; changing the shape factors in all sectors by $-20 \%$ to $20 \%$. The relative improvement of power production of the optimized layouts against the original layout for each scenario is then shown in Figure 6.

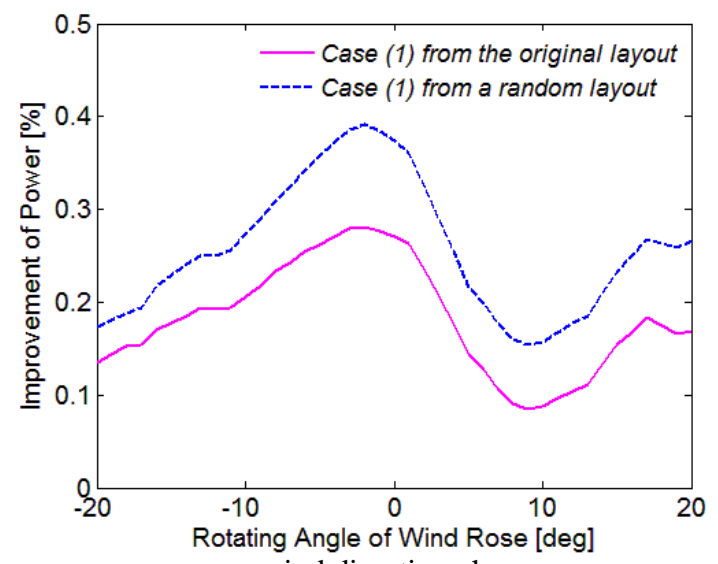

a: wind direction change

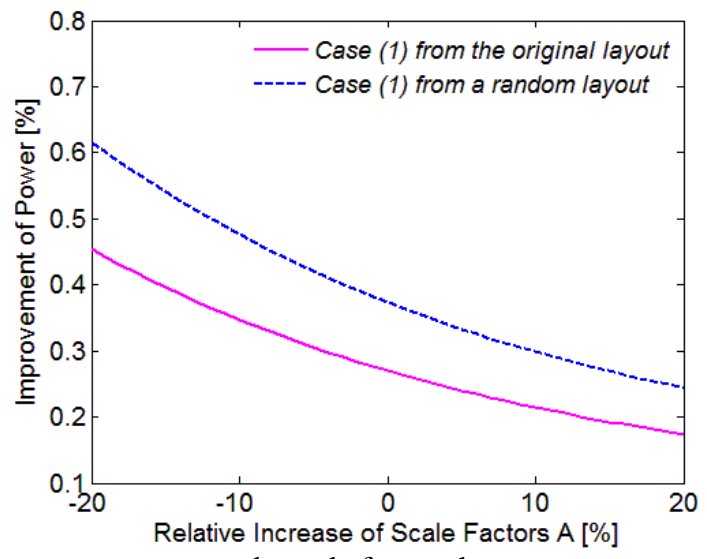

b: scale factor change

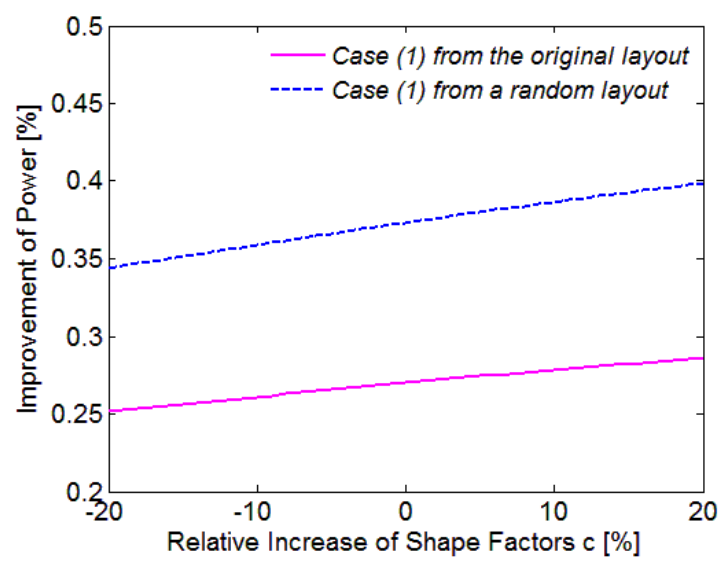

c: shape factor change

Figure 6: Improvement in power production of the optimized layouts against wind condition changes 
It can be seen from Figure 6 that the optimized layouts obtained by RS algorithm produce higher power than the original layout even when the wind conditions are changed by a significant amplitude, both in wind direction and in wind speed, and the layout obtained by using a random layout as initial layout is always better than that using the original layout as start. Thus, the robustness of the optimized layouts are quite good against wind condition changes.

An interesting fact can also be noticed in Figure $6 \mathrm{~b}$ and $6 \mathrm{c}$, that increasing the scale factors or decreasing the shape factors results in a smaller improvement of power. We can examine the wind speed distribution of the wind sector $300^{\circ}$ shown in Table 1, which has a scale factor of $A_{0}=12.17 \mathrm{~m} / \mathrm{s}$, a shape factor of $c_{0}=2.58$ and is the most powerful one in the 12 sectors. Comparing the original Weibull distribution curve and those with the changed factors, as shown in Figure 7, it can be seen that increasing the scale factor or decreasing the shape factor will increase the mean wind speed and have a higher probability of high wind speed, i.e., in the range between rated wind speed and cut-out wind speed of the WT.
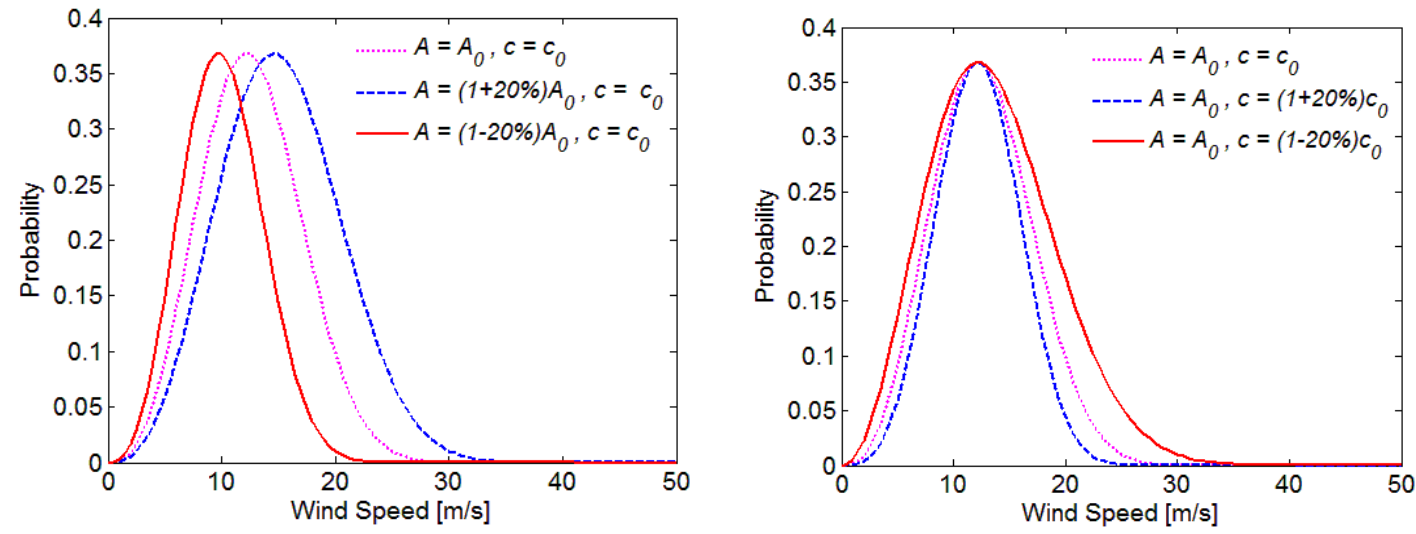

Figure 7: Different Weibull distribution curves for sector $300^{\circ}$ with different factors

It can be concluded from the typical shapes of $C_{T}$ curve and power curve as shown in Figure 2, that the wake effects between WTs and also their effects on power production are more profound in the low wind speed zone, i.e., in the range of the cut-in wind speed and the rated wind speed, but not so serious in the high wind speed zone. Therefore, stronger wind brings less wake effects and the difference between an optimized layout and a grid like layout is also relatively smaller. This fact suggests that especially for WFs built on 'poor wind' sites, the layout optimization must play an important role.

\section{Conclusions}

In this work a RS algorithm is presented to solve WF layout optimization problem. The problem formulation is presented by introducing the modeling methods for wind, wake and power production and defining the constraints and objective function. When using the RS algorithm, only one WT is moved at each time step of the optimization process. Based on this search strategy, a new evaluation schema for the WF layout is used, which saves a large amount of computation cost by reserving the wake effects data between those WTs that are not moved in the present time step.

A widely studied ideal test problem is first solved by the proposed method, and the optimized layouts produce higher power than those obtained by GA. The method is also applied to study the well-known Horns Rev 1 WF. By testing different options, an important issue regarding pre-processing the wind resource data is discovered, i.e., the number of sectors used in wind direction has a strong effect on the optimized layout. In order to obtain consistent and reliable optimization results, up to 360 sectors or more have to be used, although this will largely increase the computation cost and the results obtained are usually not so impressive as those obtained by using 12 sectors. Three cases are studied, one with the original wind resource and WF boundary, one with modified wind resource and one with 
modified WF boundary. Improvements over the original layout are obtained for all cases, and larger improvements are obtained when the WF boundary is larger or a strong single prevailing wind direction exists. Statistics of the performance in multiple runs shows a good robustness of the algorithm. Finally, the robustness analysis of the optimized layouts against wind condition changes is carried out, in order to address effects of the uncertainty in wind conditions brought by inter-annual variations in the WF's long life-time. It is found that the optimized layouts obtained by using RS algorithm show a better performance in power production than the original layout of Horn Rev 1 WF, despite of considerable variations in wind direction and speed.

The RS algorithm presented in this study exhibits several good features, such as the effectiveness of improving initial layouts in different cases, the robust performance in multiple runs and the simplicity in implementation. It can server as a useful tool for WF layout optimization. The shortage that it can only deal with WF with a fixed number of WTs will be tackled in our future study by including the number of WTs into the optimization variables. Also the issue of preprocessing of wind resource data for WF layout optimization, which has been addressed in this study, will be further investigated in future.

\section{Acknowledgment}

This work was supported by the international project (DSF Sagsnr. 10-094544) under Danish Research and Innovation Council for Strategic Research, and the Energy Technology Development and Demonstration Program (EUDP J.nr. 64013-0405) under Danish Energy Agency.

\section{References}

[1] University of Massachusetts: Wind Energy Center, "Wind energy center alumni and the early wind Industry", available at: http://www.umass.edu/windenergy/about.history.alumni.php (accessed Feb. 2013).

[2] SEAS-NVE, "Vindeby offshore wind farm", available at: http://www.seas-nve.dk/AboutSeasNve/Wind/ References/Offshore /Vindeby.aspx (accessed Feb. 2013).

[3] Z. Chen, J.M. Guerrero, F. Blaabjerg, "A review of the state of the art of power electronics for wind turbines", IEEE Transactions on Power Electronics, vol. 24(8) (2009) pp. 1859-75.

[4] L. Ma, S.Y. Luan, C.W. Jiang, H.L. Liu, Y. Zhang, "A review on the forecasting of wind speed and generated power", Renewable and Sustainable Energy Reviews, vol. 13 (2009) pp. 915-20.

[5] M. Popat, B. Wu, F.R. Liu, N. Zargari, "Coordinated control of cascaded current-source converter based offshore wind farm", IEEE Transactions on Sustainable Energy, vol. 3(3) (2012) pp. 557-65.

[6] P. Jain, Wind Energy Engineering, New York: The McGraw-Hill Companies, Inc. (2011).

[7] R.J. Templin, “An estimation of the interaction of windmills in widespread array", National Aeronautical Establishment, Laboratory Report LTR-LA-171, Otawa, Canada, (1974).

[8] P.A. Crosby, "Application of a Monte Carlo optimization technique to a cluster of wind turbines", Journal of Solar Energy Engineering, Transactions of the ASME, vol. 109 (1987) pp. 330-6.

[9] F.C. Kaminsky, R.H. Kirchhoff, “Optimal spacing of wind turbines in a wind energy power plant”, Solar Energy, vol. 39(6) (1987) pp. 467-71.

[10] M.R. Patel, Wind and Power Solar Systems, Boca Raton, FL: CRC Press (1999).

[11] K. Atias, S.P. Ladany, “Optimal economic layout of turbines on windfarms”, Wind Engineering, vol. 30(2) (2006) pp. 141-51.

[12] S. G. Mosetti, C. Poloni, B. Diviacco, "Optimization of wind turbine positioning in large windfarms by means of a genetic algorithm", Journal of Wind Engineering and Industrial Aerodynamics, vol. 51 (1994) pp. 105-16.

[13] U.A. Ozturk, B.A. Norman, "Heuristic methods for wind energy conversion system positioning", Electric Power Systems Research, vol. 70 (2004) pp. 179-85.

[14] R.A. Rivas, J. Clausen, K.S. Hansen, L.E. Jensen, "Solving the turbine positioning problem for large offshore wind 
farms by simulated annealing”, Wind Engineering, vol. 33(3) (2009) pp. 287-98.

[15] G. Marmidis, S. Lazarou, E. Pyrgioti, "Optimal placement of wind turbines in a wind park using Monte Carlo simulation", Renewable Energy, vol. 33 (2008) 1455-60.

[16] S.A. Grady, M.Y. Hussaini, M.M. Abdullah, "Placement of wind turbines using genetic algorithms", Renewable Energy, vol.30 (2005) pp. 259-270.

[17] C.Q. Wan, J. Wang, G. Yang, X. Zhang, “Optimal micro-siting of wind farms by particle swarm optimization”, Advances in Swarm Intelligence, Springer (2010) pp. 198-205.

[18] M. Wagner, J. Day, F. Neumann, “A fast and effective local search algorithm for optimizing the placement of wind turbines", Renewable Energy, vol. 51 (2013) pp. 65-70.

[19] C.N. Elkinton, J.E. Manwell, J.G. Mcgowan, “Optimizing the layout of offshore wind energy systems”, Marine Technology Society Journal, vol. 42(2) (2008) pp.19-27.

[20] P.-E. Réthoré, "State of the art in wind farm layout optimization", Wind Energy Research (2010), available at: http://windenergyresearch.org/2010/10/state-of-the-art-in-wind-farm-layout-optimization/ (accessed Feb.2013).

[21] A. Tesauro, P.-E. Réthoré, G.C. Larsen, "State of the art of wind farm optimization", Proceedings of EWEA 2012 - European Wind Energy Conference \& Exhibition, Copenhagen, Denmark, (2012).

[22] S.A. Khan, S. Rehman, "Iterative non-deterministic algorithms in on-shore wind farm design: A brief survey", Renewable and Sustainable Energy Reviews, vol. 19 (2013) pp. 370-84.

[23] J. Feng, W.Z. Shen, “Optimization of wind farm layout: A refinement method by random search", Proceedings of 2013 International Conference on Aerodynamics of Offshore Wind Energy Systems and Wakes, June 17-19, Copenhagen, Denmark (2013).

[24] J. Feng, W.Z. Shen, "Wind farm layout optimization in complex terrain: A preliminary study on a Gaussian hill", Journal of Physics: Conference Series, vol. 524(1) (2014) pp. 012146.

[25] J.F. Manwell, J.G. McGowan and A.L. Rogers, Wind Energy Explained: Theory, Design and Application (2nd edition), West Sussex: John Wiley \& Sons Ltd. (2009).

[26] I. Katic, J. Højstrup and N.O. Jensen, “A simple model for cluster efficiency”, Proceedings of the European Wind Energy Association Conference and Exhibition, Rome, Italy, (1986) pp.407-10.

[27] H.J. Gu, J. Wang, “Irregular-shape wind farm micro-siting optimization”, Energy, vol. 57 (2013) pp. 534-44.

[28] M. Gendreau, J.-Y. Potvin (Eds.), Handbook of Metaheuristics (2nd edition), Berlin: Springer (2010).

[29] L.E. Jensen, C. Mørch, P.B. Sørensen and K.H. Svendsen, "Wind measurements from the Horns Rev wind farm”, European Wind Energy Conference 2004, November 22-25, London, UK (2004).

[30] Wind Resources at Horns Rev, Report No.: D.160949, Tech-Wise A/S, Fredericia, Denmark (2002), available at: http://www.energinet.dk/SiteCollectionDocuments/Danske\%20dokumenter/Forskning\%20-\%20PSOprojekter/3248\%20Wind\%20resources\%20at\%20Horns\%20Rev.PDF

[31] F. Porté-Agel, Y.T. Wu and C.H. Chen, "A numerical study of the effects of wind direction on turbine wakes and power losses in a large wind farm", Energies, vol. 6 (2013) pp.5297-313.

\section{Appendix: Calculation of the overlapping area $\boldsymbol{A}_{o l}$}

On the transversal plane of WT $i$ 's location $\left(x=x_{i}\right)$, the rotor of WT $i$ and the wake zone of WT $j$ can be represented as two circles, with radius $R_{r}$ and $R_{i j}$ respectively, located at the same height. Depending on the transversal distance between their centres, which can be denoted as $d_{i j}=\left|y_{i}-y_{j}\right|$, the rotor of WT $i$ might be in full wake, in partial wake or out of wake of WT $j$, as shown in Figure A1. 
561

562

563

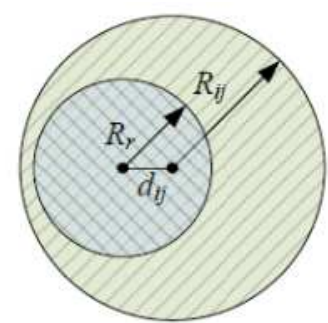

(a) $d_{i j} \leq R_{i j}-R_{r}$

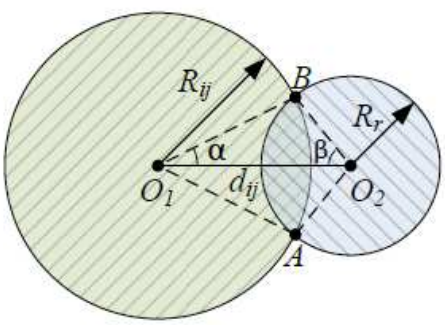

(b) $R_{i j}-R_{r}<d_{i j}<R_{i j}+R_{r}$

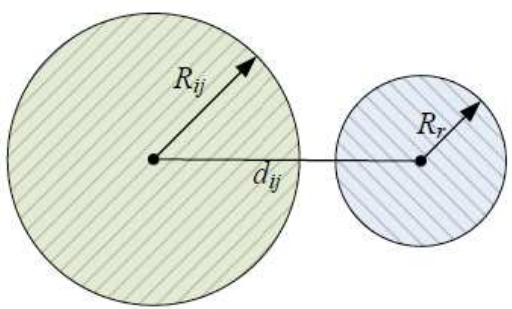

(c) $d_{i j} \geq R_{i j}+R_{r}$

Figure A1: Affected area of WT $i$ 's rotor by WT $j$ 's wake, shown as the overlapping area of the two circles, in 3 situations: (a) full wake, (b) partial wake, (c) out of wake

It is easy to see that the overlapping area of the two circles is $A_{o l}=A_{r}=\pi R_{r}^{2}$ in situation (a) and $A_{o l}=0$ in situation (c). In situation (b), the calculation requires some basic plane geometry methods.

As shown in Figure A1. (b), the overlapping area can be derived as the sum of the two circular sector areas $\left(O_{1} \widehat{A B}\right.$ and $\left.O_{2} \widehat{A B}\right)$ minusing the two triangle areas $\left(\triangle O_{1} A O_{2}\right.$ and $\left.\triangle O_{1} B O_{2}\right)$, i.e., $A_{o l}=A_{O_{1} \widehat{A B}}+A_{O_{2} \widehat{A B}}-A_{\triangle O_{1} A O_{2}}-A_{\triangle O_{1} B O_{2}}$. Noticing that the 3 edges of the triangle $O_{1} B O_{2}$ are given as $\overline{O_{1} B}=R_{i j}, \overline{O_{1} O_{2}}=d_{i j}, \overline{O_{2} B}=R_{r}$, we can easily calculate: the two angles $\alpha=\angle B O_{1} O_{2}$ and $\beta=\angle B O_{2} O_{1}$ according to the law of cosine, and the area $A_{\triangle O_{1} B O_{2}}$ using the Heron's formula. As $\triangle O_{1} A O_{2}$ and $\triangle O_{1} B O_{2}$ are congruent triangles, we can derive that $\angle B O_{1} A=2 \alpha, \angle B O_{2} A=2 \beta$, $A_{\triangle O_{1} A O_{2}}=A_{\triangle O_{1} B O_{2}}$. Based on these derivations, the overlapping area $A_{o l}$ in Figure A1 can be written as

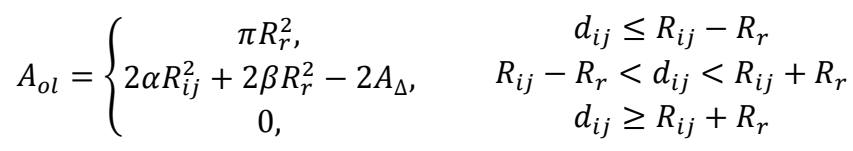

in which

$$
\alpha=\cos ^{-1}\left[\left(R_{i j}^{2}+d_{i j}^{2}-R_{r}^{2}\right) /\left(2 R_{i j} d_{i j}\right)\right], \beta=\cos ^{-1}\left[\left(R_{r}^{2}+d_{i j}^{2}-R_{i j}^{2}\right) /\left(2 R_{r} d_{i j}\right)\right],
$$

$$
A_{\Delta}=\sqrt{p\left(p-R_{i j}\right)\left(p-d_{i j}\right)\left(p-R_{r}\right)}, p=\left(R_{i j}+d_{i j}+R_{r}\right) / 2 .
$$

